\title{
Viscoelasticity of tendons under transverse compression
}

\section{Paul Buckley}

Department of Engineering Science, University of Oxford, Parks Road, Oxford OX1 3PJ, UK

paul.buckley@eng.ox.ac.uk

S. T. Samuel Salisbury

Department of Engineering Science, University of Oxford, Parks Road, Oxford OX1 3PJ, UK

sam.salisbury@oxon.org

Amy B. Zavatsky ${ }^{1}$

Department of Engineering Science, University of Oxford, Parks Road, Oxford OX1 3PJ, UK

amy.zavatsky@eng.ox.ac.uk

Accepted for publication on 15 July 2016

Copyright American Society of Mechanical Engineers (ASME)

http://biomechanical.asmedigitalcollection.asme.org/article.aspx?articleID=2543311

${ }^{1}$ Corresponding author.

BIO-16-1082 


\begin{abstract}
Tendons are highly anisotropic and also viscoelastic. For understanding and modelling their 3D deformation, information is needed on their viscoelastic response under off-axis loading. A study was made, therefore, of creep and recovery of bovine digital extensor tendons when subjected to transverse compressive stress of up to $c a 100 \mathrm{kPa}$. Pre-conditioned tendons were compression tested between glass plates, at increasing creep loads. The creep response was anomalous: the relative rate of creep reduced with increasing stress. Over each $c a 100$ s creep period, the transverse creep deformation of each tendon obeyed a power law dependence on time, with the power law exponent falling from $c a 0.18$ to an asymptote of $c a 0.058$ with increasing stress. A possible explanation is stress-driven de-hydration, as suggested previously for the similar anomalous behaviour of ligaments. Recovery after removal of each creep load was also anomalous. Relative residual strain reduced with increasing creep stress: but this is explicable in terms of the reducing relative rate of creep. When allowance was made for some adhesion occurring naturally between tendon and the glass plates, the results for a given load were consistent with creep and recovery being related through the Boltzmann Superposition Principle. The tendon tissue acted as a pressure-sensitive adhesive in contact with the glass plates: explicable in terms of the low transverse shear modulus of the tendons.
\end{abstract}

Key words: creep, recovery, de-hydration, adhesion, power law, bovine tendon, collagenous tissue, tack 


\section{Introduction}

Most previous studies of the mechanical properties of tendons focus on their response to axial tensile loading. This is understandable, since their primary physiological function is to transmit tensile force from muscle to bone. However, tendons do experience other modes of loading in-vivo, such as longitudinal tension combined with transverse compression, where a tendon wraps around a bone or is in contact with a bone near the tendon insertion. Transverse compression is potentially implicated in tendinopathy, with compression thought to be one of the mechanical stimuli driving an adaptive tissue response [1,2,3]. Moreover, tendons used in surgery as replacements for the cruciate ligaments at the knee are subject to more complex, three-dimensional loading $[4,5]$. Tendon's response in such cases cannot be deduced simply from its axial tensile response, because of the tissue's extreme anisotropy. This arises from tendon's well-known fibrous microstructure, dominated by highly hydrated collagen fibers aligned approximately parallel to the tendon axis. Tendon tissue is not only anisotropic, but also viscoelastic in its response to stress.

Three dimensional constitutive models of tendon are needed for biomechanical simulations including tendons, for example to improve understanding of healthy biomechanics, or to assist design of surgical interventions. Such models must be informed by knowledge of the 3D, time-dependent, mechanical response of tendons. However, there is no usable information yet available on the viscoelastic response of tendons under off-axis loading. The purpose of the present work is to meet this need.

Previous authors have considered some aspects of tendon viscoelasticity. The first reported study appears to be that of rat tail tendons by Rigby et al. [6], which includes measurements of tensile stress relaxation. Later work included tensile creep measurements on 
human digital extensor tendon by Cohen et al. [7] and Hooley et al. [8]. Modeling of nonlinearity of the viscoelasticity of human patellar tendon was attempted using the theory of quasi-linear viscoelasticity by Johnson et al. [9]. Duenwald et al. [10,11] studied tensile stress relaxation of porcine digital flexor tendons, and compared it to that of porcine medial collateral ligaments. Their work included study of the nonlinearity of the viscoelasticity: the strain dependence of stress relaxation. This aspect was further studied in mouse patellar tendons by Dourte et al. [12] and in rat tail tendon fascicles by Davies and De Vita [13] and LaCroix et al. [14]. In all these previous studies of creep or stress relaxation in tendons, however, the tensile stress or strain was applied in the fiber-aligned direction.

There have also been studies of tendon deformation under transverse stress. Some authors studied inhomogeneous localized transverse compression of tendons, using an indentor [12,15,16]. However Lynch et al. [17] and Williams et al. [18] managed to study nominally uniform tensile and compressive deformations respectively, in specimens cut from sheep flexor tendons and rabbit patellar tendons respectively. More recently, Main et al. studied the transverse compression of whole human digital flexor tendons [19], although with a technique that produced intrinsically non-uniform deformation. However, none of these studies considered time-dependence of the response.

The present paper reports results from the second part of an experimental study of deformation in bovine digital extensor tendons, compressed transverse to their fiber-aligned axes. In the first part of the study [20], isochronal measurements of deformation were combined with hyper-elastic finite element simulations of the inhomogeneous deformation, to determine values of the two apparent elastic constants governing deformation purely in the transverse (isotropic) plane. Here, in the second part, time-dependence of the deformation is examined. The 
loading profile consisted of a pre-conditioning sequence followed by a series of load-on + loadoff cycles at increasing levels of transverse compressive load. Tendons exhibited significant creep during the load-on, and recovery during the load-off, portions of the loading cycle. This is believed to be the first attempt to characterize the viscoelasticity of tendon under transverse stress. The aim here is to measure the time-dependence and stress-dependence of the response. In particular, the data are tested to assess their adherence to the theories of linear viscoelasticity, and of quasi-linear viscoelasticity (QLV).

\section{Materials and Methods}

Descriptions of the experimental equipment and protocol are given by Salisbury et al. [20], to which the reader should refer for more information. Only essential details are given here.

The tendons tested were typically $c a 160 \mathrm{~mm}$ in length. They were tested subjected to transverse compression using a custom-built test rig, also allowing in-situ image-based measurement of the tendon's cross-sectional shape and area, using a method described previously [21]. The tendon was initially suspended vertically under its own weight from a drill chuck acting as upper grip, with a length of 15-20 mm in the chuck. During each creep phase of the testing, the tendon was compressed horizontally over a length of $40 \mathrm{~mm}$, approximately 70 mm distant from the chuck, between two flat, parallel, rigid (relative to the tendon) glass plates under a prescribed force $F$.

One of the glass plates was free to approach or move away from the other, as the tendon crept or recovered, while an LVDT displacement transducer monitored this relative movement. Simultaneously, a digital video camera recorded an image of the tendon through one of the glass plates (optically flat) in the direction of loading, allowing observation of deformation in the 
plane normal to the loading direction. There was some expansion in the direction transverse to the fiber axis, but no noticeable deformation in the axial direction - see the account given previously [20]. After pre-conditioning in compression (see below), all tendons had an approximately race-track shaped cross-section [20]. This representative shape is shown schematically in Figure 1. The origin $\mathrm{O}$ is defined at the centroid of the representative crosssection, and co-ordinate axes $\mathrm{O} x, \mathrm{O} y$ are defined parallel and perpendicular to the major and minor principal axes of the race-track respectively, as shown in Figure 1. The $\mathrm{O} z$ axis is parallel to the long axis of the tendon. The LVDT monitored the thickness of the tendon $\Delta y$, while the video image gave the width $\Delta x$ at the center of the compressed zone. In the previous report from Salisbury et al. [20], deformations in both $x$ and $y$ directions were exploited. Here, only the $\Delta y$ information is exploited, since its time-dependence was much better resolved experimentally, than that of $\Delta x$.

From the shape in Figure 1, it is clear that strain and stress within the tendon cannot be homogeneous. Also there were differences in cross-sectional dimensions between the tendons. Therefore to compare objectively the responses of different tendons, we represent the applied loads and displacements in normalized form as mean values of stress and strain, averaged along the two axes of the cross-section $\mathrm{AB}$ and $\mathrm{CD}$, respectively. Mirror symmetry of the cross-section and of the loading conditions about the planes defined by $\mathrm{O} z$ and $\mathrm{AB}$, and $\mathrm{O} z$ and $\mathrm{CD}$, dictates that in these planes the axes $x, y, z$ are principal directions, labeled here 1,2,3 respectively. Therefore change in thickness is represented here as the principal nominal strain $\varepsilon_{2}$ averaged along the $y$-direction line CD: $\left\langle\varepsilon_{2}\right\rangle_{y}$. Similarly, the applied stress is represented by the principal nominal stress $\sigma_{2}$, averaged along the $x$-direction line AB: $\left\langle\sigma_{2}\right\rangle_{x}$. Hence, with subscript 0 
indicating initial values prior to application of the creep load and superscripts $c$ and $r$ referring to the creep and recovery phase of each creep/recovery test:

$$
\begin{gathered}
\left\langle\varepsilon_{2}\right\rangle_{y}=\frac{\Delta y}{\Delta y_{0}}-1 ; \\
\text { during creep }\left\langle\sigma_{2}\right\rangle_{x}=\left\langle\sigma_{2}\right\rangle_{x}^{c} \equiv-\frac{F}{L \Delta x_{0}} ; \quad \text { during recovery }\left\langle\sigma_{2}\right\rangle_{x}=\left\langle\sigma_{2}\right\rangle_{x}^{r} \equiv 0
\end{gathered}
$$

where the length of the compressed zone in direction $\mathrm{O} z$ is $L(=40 \mathrm{~mm})$.

Seven tendons were included in the main study. They were bovine digital extensor tendons, dissected from bovine feet all from the same batch of animals, and stored in a freezer at $-18^{\circ} \mathrm{C}$ soon after slaughter until the day before testing. The age of the animals was $18-30$ months. The tendons were allowed to return to room temperature overnight while soaked in $10 \%$ sucrose solution, in order to return the tendons to a level of hydration as close as possible to that of in-vivo conditions [22]. They were rinsed with phosphate-buffered saline (PBS) immediately prior to testing, at $c a 20^{\circ} \mathrm{C}$, to wash away any surface residue of sucrose. After mounting in the test rig, each tendon was first pre-conditioned with ten load-on + load-off cycles of $20.8 \mathrm{~N}$ transverse compressive load: $10 \mathrm{~s}$ on $+10 \mathrm{~s}$ off. The cross-sectional area and shape of the preconditioned tendon were then measured at five locations along its axis, in the test region, from 1 $\mathrm{mm}$ above to $1 \mathrm{~mm}$ below the glass window, using the method of Salisbury et al. [21]. The initial cross-section widths and heights of the various tendons in the center of the compressed region, after pre-conditioning, ranged as follows: $\Delta x_{0}=8.9-15.6 \mathrm{~mm}, \Delta y_{0}=3.7-5.8 \mathrm{~mm}$.

Following the cross-section measurement, the tendon was soaked in $10 \%$ sucrose again for 1 hour, and again rinsed in PBS. The main test sequence followed, in which ten compressive creep/recovery load profiles were applied, with loads increasing from $0.9 \mathrm{~N}$ to $39.7 \mathrm{~N}(0.9 \mathrm{~N}, 2.3$ $\mathrm{N}, 3.8 \mathrm{~N}, 5.7 \mathrm{~N}, 8.5 \mathrm{~N}, 12.3 \mathrm{~N}, 17.0 \mathrm{~N}, 22.7 \mathrm{~N}, 30.2 \mathrm{~N}, 39.7 \mathrm{~N}$, with load steps chosen to provide 
approximately evenly spaced displacement versus time curves). Each profile consisted of: (a) application of a pre-load of $0.9 \mathrm{~N}$ for $5 \mathrm{~s}$, followed by unloading and $50 \mathrm{~s}$ recovery; and then (b) application of the main creep load for $120 \mathrm{~s}$, followed by unloading and $660 \mathrm{~s}$ of recovery, while the deformation was monitored. The time taken to apply or remove each creep load was up to $c a$ 1s. Between each profile, the tendon was allowed to recover for one hour under no load in the bath of sucrose solution, and then rinsed in PBS. This step was assumed to return the tendon to its original state of hydration and to allow it to recover its original dimensions. The image-based measurements of tendon widths in the x-direction were used to quantify the accuracy of the latter assumption. Following the one-hour soaks, percentage deviations of tendon widths from their original values (i.e. values immediately after pre-conditioning) were found to be only $1.9 \% \pm$ $2.7 \%$ (mean \pm standard deviation over 63 experiments).

A further three tendons from the same batch, prepared in the same manner, were preconditioned and tested similarly, but with six compressive creep/recovery load profiles in the sequence: $2.8 \mathrm{~N}, 20.8 \mathrm{~N}, 39.7 \mathrm{~N}, 2.8 \mathrm{~N}, 20.8 \mathrm{~N}, 39.7 \mathrm{~N}$ to determine the repeatability of the displacement measurements at a given load, for a given tendon. Further details were given previously [20,23]. For example, the displacement was found to be repeatable, at the $95 \%$ confidence level, to within $\pm 0.05 \mathrm{~mm}$ for a creep time of $100 \mathrm{~s}$. Further measures of repeatability are given below.

There was apparent 'friction' between the glass plates and the tendons. The apparent coefficient of static friction $\mu_{\mathrm{s}}$ was found to be $0.35 \pm 0.01$ [20], and this value was used in the finite element modelling reported in Salisbury et al. [20]. We show below that the physical origin of this apparent 'friction' appears actually to be a form of adhesion between the tendon and the glass plates. 


\section{Results}

All tendons revealed time-dependent creep and recovery. Typical data for one tendon are given in Figure 2, which shows strain versus time for each level of creep stress $\left\langle\sigma_{2}\right\rangle_{x}^{c}$. All the tendons tested showed strain-time curves similar to those of Figure 2. Moreover, a similar pattern of creep and recovery strain (but of opposite sign) was also seen in the $x$ direction (see the data presented previously [20]). Figure 2 shows that the strain recovered only partially when the creep stress was removed, on the time-scale of the present recovery measurements. A distinctive feature is that the relative recovery of strain appears least complete at the lowest stresses. It is especially clear at the lowest stresses that recovery has ceased and there remains a permanent residual strain.

The $y$ direction displacement was sufficiently well resolved to allow quantitative analysis of its time-dependence. Thus data were collected at $100 \mathrm{~Hz}$ and rms noise on the displacement data was below $1 \mu \mathrm{m}$. Although the creep is clearly nonlinear viscoelastic - see evidence discussed below - creep curves were examined to check whether they exhibited evidence for a simplification in the nonlinearity: separable dependence of the strain on time and stress. This would mean the averaged strain at creep time $t$, for a particular value of applied creep stress $\left\langle\sigma_{2}\right\rangle_{x}^{c}$, is expressible in terms of two separable functions $g$ and $h$, thus

$$
\left\langle\varepsilon_{2}\right\rangle_{y}=h\left(\left\langle\sigma_{2}\right\rangle_{x}^{c}\right) g(t) \text { hence } \log _{10}\left\langle\varepsilon_{2}\right\rangle_{y}=\log _{10} h\left(\left\langle\sigma_{2}\right\rangle_{x}^{c}\right)+\log _{10} g(t)
$$

This would correspond to the analogue (for strain in terms of stress) of the quasi-linear viscoelastic (QLV) model proposed (for stress in terms of strain) by Fung [24]. The applicability 
of equation (2) was tested following the procedure used by Provenzano [25] to examine axial tensile creep and stress relaxation in rat ligaments.

For each tendon, its whole series of creep curves (expressed as strain versus time) was plotted on a single double logarithmic graph. A typical graph for one tendon is shown in Figure 3. There is a systematic tendency for the gradient to decrease with increasing applied stress. Thus the curves do not superpose under pure vertical shifts as required by the second of equations (2): the curves do not show separable dependence on time and stress. The same was true for all the tendons tested.

As seen in Figure 3, however, the creep curves are highly linear for the range of creep times shown on this double log plot. Thus for this time-scale they can all be approximated well by power laws in time, where isochronal relative rates of creep are proportional to the power law exponent $n$ :

$$
\log _{10}\left\langle\varepsilon_{2}\right\rangle_{y}=\log _{10} A+n \log _{10} t \text { hence }\left\langle\varepsilon_{2}\right\rangle_{y}=A t^{n} \text { and } \frac{\left\langle\dot{\varepsilon}_{2}\right\rangle_{y}}{\left\langle\varepsilon_{2}\right\rangle_{y}}=\frac{n}{t}
$$

where $A$ is independent of time (and is the averaged isochronal strain $\left\langle\varepsilon_{2}\right\rangle_{y}$ at a creep time of 1 s). This relationship was found to apply to all creep tests on all the tendons tested. Thus, for all creep tests conducted on the seven tendons of the main study, the measured $y$ direction averaged creep strain obeyed a power law dependence on time. Linear regressions were fitted to the double logarithmic plots over the creep time interval $10 \mathrm{~s}-100 \mathrm{~s}$, yielding correlation coefficients (mean \pm standard deviation) of $R^{2}=0.999 \pm 0.001$ and values for the coefficient $A$ and exponent $n$. (Creep times of less than $10 \mathrm{~s}$ were neglected in the linear regressions, because of corruption of the creep data arising from the stress not having been applied instantaneously.) 
Although the power law fitted each individual creep test well, non-linearity of the viscoelasticity is revealed by how coefficients $A$ and $n$ varied with applied creep stress. Linearity would require $A$ to be linear in the stress and $n$ to be independent of stress. Figure 4 shows the relation between $A$ and the creep stress $\left\langle\sigma_{2}\right\rangle_{x}^{c}$ for all the tendons. The nonlinearity is clear: all tendons show stiffening with increasing stress, with the values of stiffness (inverse of the gradient) varying between the tendons.

Figure 3 shows that $n$ (the gradient) also varies with stress, revealing non-compliance with equations (2). Specifically, the exponent $n$ falls with increasing stress. This observation is quantified in Figure 5, where values of $n$ are collected for all stress levels and all tendons, and are plotted versus the applied creep stress. The graph reveals a systematic tendency for $n$ to decrease from a value of $c a 0.18$ to an asymptotic level of $c a 0.06$ with increasing applied stress. An empirical description of this tendency is provided by a decaying exponential function. The 'least-squares' best fit of the data to such a function, included as the line in Figure 5, is the expression

$$
n=0.058+0.120 \exp \left(-0.084\left\langle\sigma_{2}\right\rangle_{x}^{c}\right)
$$

where stress is expressed in $\mathrm{kPa}$. There is significant scatter: rms deviation of the data from equation (4) is 0.028 . However, equation (4) indicates unambiguously a rapid reduction of $n$ with increasing load: the exponential decay is $99 \%$ complete within a stress of only ca $55 \mathrm{kPa}$. Moreover, results of the extra set of creep tests, designed to assess repeatability, showed that measurements of $n$ (for a given tendon and load level) were repeatable to within 0.026 at the 95\% confidence level, and only slightly dependent on the order of measurement: the mean value of the difference second-minus-first measurement of $n$ was only -0.0015 . Both these figures are small compared to the drop of 0.120 in $n$ given by equation (4). This confirms that the drop is a BIO-16-1082 
genuine feature of tendon creep behaviour with varying load level, and not merely a consequence of the particular order of testing used in the experiments (in which creep tests were conducted at increasing values of load).

Incomplete recovery in the $y$ direction was quantified in terms of the relative residual strain $\left\langle\varepsilon_{2}\right\rangle_{y} /\left\langle\varepsilon_{2}\right\rangle_{y}^{\max }$ remaining at any particular recovery time, where $\left\langle\varepsilon_{2}\right\rangle_{y}^{\max }$ is the maximum averaged creep strain, just prior to removal of the creep stress. Relative residual strain at a recovery time $5 t_{\mathrm{u}}$, where $t_{\mathrm{u}}$ is the creep time at unloading ( $c a 120 \mathrm{~s}$ ), is plotted in Figure 6 versus the applied creep stress for all tendons. This reveals a significant decrease in relative residual strain with increasing creep stress, although the magnitude of the decrease differs between tendons, varying from $c a 0.2$ to $c a 0.4$. Again the repeatability tests confirmed the significance of this result. They showed that measurements of $\left\langle\varepsilon_{2}\right\rangle_{y} /\left\langle\varepsilon_{2}\right\rangle_{y}^{\max }$ were repeatable to 0.097 at the $95 \%$ confidence level, and were only slightly dependent on the order of measurement: the mean value of the second-minus-first measurement of $\left\langle\varepsilon_{2}\right\rangle_{y} /\left\langle\varepsilon_{2}\right\rangle_{y}^{\max }$ was -0.0083 . Hence the reduction of relative residual strain with increase in creep load seen in Figure 6 is a genuine feature of the tendons, and not a consequence of the particular order of testing.

\section{Discussion}

\subsection{Creep}

The stress-dependent stiffening seen in isochronal creep strains (Figure 4) can be explained as primarily a nonlinearity of geometrical origin. This was demonstrated previously, in finite element simulations of the experiments, using a linear hyper-elastic constitutive model for tendon tissue [20] - see further comments below in Section 4.2. At the strain levels reached in the present experiments, on application of the creep load there is significant contraction in the $y$ BIO-16-1082 
direction and expansion in the $x$ direction. Hence $\left\langle\varepsilon_{2}\right\rangle_{y}$ is less than the corresponding true strain, and $\left\langle\sigma_{2}\right\rangle_{x}$ is greater than the corresponding true stress.

The most significant feature of the creep data is the pronounced reduction in relative rate of creep (and hence $n$ ) with increase in applied stress. Reports of similar behaviour in tendons are rare in the literature. Dourte et al., in a study of mouse patellar tendons, observed a reduction in relative rate of axial stress relaxation with increase in strain [12]. In a study of the effects of age on axial tensile stress relaxation in rat tail tendons, LaCroix et al. showed a single data point, for tendons from the youngest animals (3 months) at the lowest applied strain (0.005) where the relative relaxation rate reduced with increasing strain [14]. But these results contrast with all the data of LaCroix et al. at higher strains, and all other published measurements of tendon viscoelasticity of which the authors are aware. In all these other cases, the relative rate of creep/relaxation observed was either independent of stress/strain, consistent with QLV (see Cohen et al. [7] and Elliott et al. [26]) or to increase with stress/strain, as found by Salisbury for axial tensile testing of tendons from the same batch as those of the present study [23], by Duenwald et al. [11] and Davis and De Vita [13]. (Although not discussed in their paper, the data of Cohen et al. for axial tensile creep of human digital extensor tendon for strains up to $1.7 \%$ can be shown to follow the power law as in equations (2), but with constant $n=0.044 \pm 0.002$ ). However, the reader will recall that, in all these previous studies of tendon viscoelasticity, loads were applied along the fiber-aligned direction.

Non-linearity of this usual form is also typical of the viscoelasticity of all synthetic polymers. Moreover, it is readily explained in terms of stress-induced shortening of relaxation times, as predicted by all physically-based constitutive models of polymer viscoelasticity based on Eyring kinetics - e.g. see Buckley and Jones [27]. 
Clearly therefore, a reduction in relative rate of creep/relaxation with increase in stress/strain, as seen here for transverse compression of tendons, is anomalous. It cannot be explained in terms of current physically-based constitutive models for polymers. However Lakes, Vanderby and co-workers have shown that this anomalous form of nonlinear viscoelasticity is regularly observed in ligaments. They observed it in axial tensile viscoelastic tests on medial collateral ligaments (MCLs) from rats [25], from rabbits [28] and from pigs [11]. It was also seen by Bonafasi-Lista et al. in stress relaxation tests on human MCL when subject to axial tension, transverse tension or axial shear [29].

The most plausible explanation offered so far for this anomalous behaviour was offered by Provenzano et al. for ligaments [25]: the application of stress squeezes out some water, as has been observed previously for tensile loading of tendons - see Hannafin and Arnoczky [30] and Han et al. [31]. Reduced hydration is known to decrease the relative rate of stress relaxation in tendons [32] and ligaments [22]. The reason is most likely to be that movement of free water within the tissue is the origin of the viscoelasticity. In addition, similar to synthetic polymers containing water bound to hydrophilic chemical groups (e.g. the polyamides), reduction in bound water content reduces molecular mobility and hence also influences (lengthens) viscoelastic relaxation times.

Why this effect is more visible in ligaments than in tendons, and more in transverse compression of tendons than in axial extension of them, is not known for certain at the present time, particularly since a number of factors can potentially affect the movement of water in these tissues. Firstly, the amount of water in ligaments and tendons can be anywhere from $60-80 \%$ of the extracellular matrix [33], and the distribution of this water throughout the tissue is unlikely to be uniform. Secondly, the apparent diffusion coefficient of water in tendon, and presumably also 
in ligament, displays both directional anisotropy (parallel versus perpendicular to the main fiber direction) [31,34] and inhomogeneity (surface versus core) [35] and can be affected by the choice of solution used for tissue storage [31]. Thirdly, the fibril directions and packing within the tissue are likely to dictate the path (and hence the "tortuosity") associated with water movement [34]; these features are different in tendon and ligament (fibrils being less regularly arranged in ligament [36]) and vary with the location and function of the tissue in the body. Finally, the direction, amount, and type of any applied load will also be critical. The present compression tests provide a macroscopic pressure gradient between the interior of the tendon and its free surface, and this will naturally tend to expel free water, if there are available pathways through the microstructure. In other tests the nature of the mechanism is much less clear.

Given that each experimental profile consisted of a sequence of creep/recovery tests at increasing load levels, invoking stress-induced de-hydration to explain the creep anomaly raises the question: what is the state of hydration at the start of each creep test? The assumption made here is that the one hour soak in $10 \%$ sucrose solution under no load between tests was sufficient to fully re-hydrate the tendon, before the start of the next test. This is supported by the results of the repeatability tests.

Of course it is possible that the use of a liquid bath would have produced different results. If the suggestion made in this paper is correct - application of a transverse compressive load causes some de-hydration of tendon - then use of an aqueous liquid environment would be expected to cause the tendon to gradually re-hydrate upon unloading. However, this would be a slower process than the stress-driven de-hydration (there would be no pressure gradient to assist it), and the resulting recovery data would then be impossible to interpret quantitatively because of the simultaneously varying degree of hydration. Our use of a dry environment avoids this BIO-16-1082 
complication, although with the potential complication of water loss by evaporation from the tendon's surface. But, using published values for the apparent diffusion coefficient of water in tendon [31], it can be shown that during the time-scale of a single creep/recovery experiment (ca 14 minutes) the change of water content would be negligible: e.g. at $1 \mathrm{~mm}$ from the surface the water content would change by only $\mathrm{ca} 1 \%$. It could be a fruitful objective for future work to compare the response of tendon to transverse loads with and without a liquid environment. But this lay beyond the scope of the present experiments.

\subsection{Recovery}

The reduction in relative residual strain with increasing creep stress, clearly evident in Figures 2 and 6, is also anomalous. The opposite trend is expected, since increasing stress is expected to cause the gradual appearance of apparently permanent plastic deformation and hence an increase in relative residual strain. However, the recovery anomaly seen here can be explained qualitatively as follows. For a material that creeps according to equations (3), for which the only stress acting along $\mathrm{CD}$ during creep is a constant $\sigma_{2}$, which is absent during recovery, the Boltzmann Superposition Principle (BSP) of linear viscoelasticity predicts the relative residual strain remaining at time $t\left(>t_{\mathrm{u}}\right)$ will be

$$
\frac{\left\langle\varepsilon_{2}\right\rangle_{y}}{\left\langle\varepsilon_{2}\right\rangle_{y}^{\max }}=\left(\frac{t}{t_{\mathrm{u}}}\right)^{n}-\left(\frac{t-t_{\mathrm{u}}}{t_{\mathrm{u}}}\right)^{n}
$$

For given values of $t$ and $t_{\mathrm{u}}$, the predicted relative residual strain is sensitive to $n$ : increasing with increase in $n$. This explains the similarity of form between Figures 5 and 6 . Thus the anomalous 
trend in the present recovery data is explained qualitatively by the anomalous trend seen in the present creep data.

Nevertheless, this alone does not provide a quantitative explanation of the recovery data. Substituting into equation (5) the maximum and minimum values of $n$ given by equation (4), yields values for the expected relative residual strain of 0.044 and 0.012 , respectively, for a recovery time $t-t_{\mathrm{u}}=5 t_{\mathrm{u}}$ as applying to Figure 6. The observed values of relative residual strain seen in Figure 6 (0.2 or greater) are clearly much greater than this, indicating that equation (5) is not an accurate representation. Does this indicate deviation from the BSP, and hence that the recovery response is non-linear viscoelastic?

The low value of tendon shear modulus $G \approx 100 \mathrm{kPa}$ in the transverse plane [20] is a warning that inconsistency of the data with equation (5) may not indicate deviation from the BSP, but instead may be an experimental artefact, caused by adhesion between the tendon and the compression plates. It is known in the field of pressure-sensitive adhesives (PSA) that a modulus as low as this, in a material pressed against a dry rigid surface, leads to a degree of adhesive bonding, or 'tack', between them. The reason is conformability of the material leading to a high degree of intimate molecular-level contact (i.e. wetting of the surface), and hence of van der Waals bonding, between them. Moreover, provided the modulus does not lie far below this, the tack increases with increase in the pressure applied, as the proportion of 'contact' area that is in molecular-level contact increases. Creton and Leibler provide further discussion of this phenomenon [37]. Such adhesion resists not only separation of the surfaces, but also the relative sliding of them.

The Appendix quantifies the effect such adhesion would have on the recovery data, and suggests a means to test whether it is the cause of disagreement with equation (5). Specifically, BIO-16-1082 
adhesion at the glass-tendon interface would give rise to an $x$-direction transverse stress ('tack stress') in planes $\mathrm{AB}$ and $\mathrm{CD}$ of the cross-section, which is neglected in equation (5). Let its value averaged along $\mathrm{AB}$ be $\left\langle\sigma_{1}\right\rangle_{x}$.

This stress was deduced from the recovery data, for each creep/recovery experiment, using the method described in the Appendix. This allowed the hypothesis of pressure-sensitive adhesion to be tested, by examining the relation between $\left\langle\sigma_{1}\right\rangle_{x}$ and the applied creep stress $\left\langle\sigma_{2}\right\rangle_{x}^{c}$, for all seven tendons. Figure 7 shows typical results for four tendons. As illustrated here, all tendons showed a highly linear relationship between the apparent tack stress and the applied creep stress:

$$
\left\langle\sigma_{1}\right\rangle_{x}=k\left\langle\sigma_{2}\right\rangle_{x}
$$

The mean \pm standard deviation (SD) of the correlation coefficients over all seven tendons was $R^{2}$ $=0.992 \pm 0.006$. The mean $\pm \mathrm{SD}$ value of the gradient was $k=0.216 \pm 0.041$. This is precisely the form of relationship expected for pressure-sensitive adhesion [37]. Thus these data support the hypothesis that pressure-sensitive adhesion is the cause of recovery being less complete than predicted by equation (5). There is no need to invoke departure from the BSP.

As a further check, the first of equations (A3) was used to compute the expected residual strain $\left\langle\varepsilon_{2}\right\rangle_{y}$ versus time during recovery, for comparison with experimental data. Typical comparisons are shown for the particular tendon of Figures 2 and 3 in Figure 8 . As can be seen, the time-dependence of recovery was captured well for the lowest creep stresses, but the residual strain was increasingly under-predicted at short recovery times for the highest creep loads. The most likely explanation for the discrepancy at short times and high stresses is that equation (A3) neglects the tensile $y$ direction tack stress expected to resist separation of the tendon and glass surfaces, as the cross-section recovers towards its original shape with a smaller contact area. This BIO-16-1082 
geometrical effect was neglected in the analysis in the Appendix, and is significant only at finite deformations and short recovery times, where significant geometry change is occurring during recovery.

\section{Conclusions}

The work described here has demonstrated a method for studying the viscoelasticity of tendons when compressed transverse to their fiber-aligned axes. Tendons were compressed transversely between flat glass plates. Each of a set of bovine digital extensor tendons, all from the same source, was subject at $c a 20^{\circ} \mathrm{C}$ to pre-conditioning load/unload cycles, and then to a set of ten creep/recovery load profiles at increasing loads, with an hour of relaxation under no load in $10 \%$ sucrose solution between each profile.

The creep/recovery response was found to be non-linear viscoelastic, but not consistent with QLV. Several aspects of the viscoelasticity are anomalous. Firstly, the isochronal transverse response of the tendons shows strain-induced stiffening. However, this is primarily a geometrical effect for the strain levels encountered in the present work. Finite element modelling of the isochronal deformation, using a hyper-elastic constitutive model, showed previously that such stiffening is expected from change of cross-section shape with increasing transverse compressive load [20].

Secondly, the relative rate of transverse compressive creep falls with increase in stress. This is counter to almost all previous reports of tendon viscoelasticity (all of which concerned axial tensile deformation). It is unknown in synthetic polymers, and is inconsistent with widely invoked physical models for the origin of nonlinear viscoelasticity in polymers. It has, however, been reported previously in ligaments, under various loading conditions. The most promising 
explanation is stress-induced de-hydration. Reduced water content is known to reduce the degree of time-dependence in deformation of tendons and ligaments. The new evidence presented here shows that the mechanical effects of stress-induced de-hydration are visible when tendons are compressed in the transverse direction, although the literature shows they are usually not visible when tendons are loaded in the fiber-aligned direction.

Thirdly, the recovery on removal of load is anomalous: the relative residual strain falls with increase in creep stress: the opposite tendency to that observed normally in other materials. However, this is a natural consequence of the fall in the relative rate of creep with increasing stress, when combined with the Boltzmann Superposition Principle (BSP).

Fourthly, however, the recovery appears not to comply quantitatively with the BSP, as expressed by equation (5). The extent of recovery appears always to be lower than expected according to the BSP, implying that viscoelasticity of the tendons is nonlinear even at the lowest creep stresses. However, further analysis of the data has revealed this to be an experimental artefact. It arises because the tendon in transverse compression has such a low stiffness that it behaves as a pressure sensitive adhesive when pressed against glass plates. Adhesion gives rise to a tack stress resisting displacements in the plane normal to the applied load, during both creep and recovery, neglected in equation (5). When quantified, this tack stress is found to be proportional to the applied stress in every tendon, verifying the description in terms of pressure sensitive adhesion, without the need to invoke departure from the BSP.

In summary, allowing for the effects of this adhesion, the present data are consistent with the following description. Under transverse compression, tendon tissue shows anomalous creep and recovery because the creep stress causes some de-hydration. But the tendon's response at a 
given level of hydration is consistent with linear viscoelasticity, for levels of transverse stress applied in the present work: up to $c a 100 \mathrm{kPa}$.

\section{Appendix: Tendon/glass adhesion restricts creep and recovery}

We propose a two-part hypothesis to explain, quantitatively, the magnitude of incomplete recovery in the presence of pressure-sensitive adhesion between the tendon and the glass compression plates. (1) When each compressive creep stress $\left\langle\sigma_{2}\right\rangle_{x}^{c}$ is applied, it is assumed to establish throughout the tendon (caused by some de-hydration) a new power law exponent $n$, and by change of shape (possibly with a contribution from the de-hydration) a new power law coefficient $A$; but thereafter the tendon behaves as a linear viscoelastic solid. (2) In addition, the tendon is assumed to act as a PSA in its contact with the glass plates. Hence, when they are pressed together, some adhesion is established at the tendon-glass interfaces. Any $x$ direction sliding of the tendon is then resisted by a shear stress $\sigma_{x y}$ at the interfaces, that scales with the fractional area of true contact. (This shear stress should not be confused with friction. It is the stress required to sever van der Waals bonds at the interface. Its magnitude is therefore determined by the fractional area of contact. If contact remains after the normal stress is removed, the shear resistance remains.) Such a shear stress must translate (via the requirement for $x$ direction equilibrium) into a transverse direct stress $\sigma_{x x}(x, y)$. The effect may be quantified as follows.

We recognise the tendon as being transversely isotropic and approximately incompressible [20], and within the compression zone as being subject to approximately plane BIO-16-1082 
strain conditions [20]. Moreover, since the response is assumed to be linear viscoelastic, in these creep/recovery tests we expect (from the elastic-viscoelastic correspondence principle) the local stresses to be constant [20] during creep or recovery. Invoking also the observed power law timedependence (see above), we can write the $y$ direction direct strain at any point $(x, y, z)$ within the compression zone during creep in the form

$$
\varepsilon_{y y}=\frac{1}{4} J_{1} t^{n}\left[\sigma_{y y}-\sigma_{x x}\right]
$$

where the parameters $n$ (and possibly $J_{1}$ ) are functions of the local stress: $J_{1}$ is the corresponding 1 second shear creep compliance.

We now approximate each tendon cross-section as having the representative race-track shaped geometry introduced earlier (Figure 1), and we average equation (A1) along the centreline in the $x$ direction: AB. Again invoking incompressibility and elastic-viscoelastic correspondence, the strain distribution is expected to be constant during creep and recovery, merely scaling with time. Therefore, at all times, a constant dimensionless factor $f$ relates $\left\langle\varepsilon_{2}\right\rangle_{y}$, averaged along $\mathrm{CD}$, to the same principal strain averaged along $\mathrm{AB}:\left\langle\varepsilon_{2}\right\rangle_{x}$. Consequently during creep $\left(t<t_{\mathrm{u}}\right)$

$$
\left\langle\varepsilon_{2}\right\rangle_{y}=f\left\langle\varepsilon_{2}\right\rangle_{x}=\frac{1}{4} f J_{1} t^{n}\left[\left\langle\sigma_{2}\right\rangle_{x}^{c}-\left\langle\sigma_{1}\right\rangle_{x}^{c}\right]
$$

The factor $f$ and the magnitude of creep 'tack' stress $\left\langle\sigma_{1}\right\rangle_{x}^{c}$ are assumed here to depend uniquely on the applied creep stress $\left\langle\sigma_{2}\right\rangle_{x}^{c}$, i.e. they are constant from the moment the creep stress is applied. During recovery, the tendon remains in contact with the glass plates but the direction of $x$ displacements and hence the sign of $\left\langle\sigma_{1}\right\rangle_{x}$ are reversed, but we assume the 
adhesion remains and the magnitude of this stress remains unchanged. Thus we expect $\left\langle\sigma_{1}\right\rangle_{x}^{r}=$ $-\left\langle\sigma_{1}\right\rangle_{x}^{c}$. Extending equation (A2) to include recovery by applying the BSP, then allows us to predict the relative residual strain during recovery $\left(t>t_{\mathrm{u}}\right)$

$$
\frac{\left\langle\varepsilon_{2}\right\rangle_{y}}{\left\langle\varepsilon_{2}\right\rangle_{y}^{\max }}=\left(\frac{t}{t_{\mathrm{u}}}\right)^{n}-\Phi\left(\frac{t-t_{\mathrm{u}}}{t_{\mathrm{u}}}\right)^{n} \text { where } \Phi=\frac{\left\langle\sigma_{2}\right\rangle_{x}^{c}-2\left\langle\sigma_{1}\right\rangle_{x}^{c}}{\left\langle\sigma_{2}\right\rangle_{x}^{c}-\left\langle\sigma_{1}\right\rangle_{x}^{c}}
$$

The parameter $\Phi$ quantifies the freedom the tendon has to slide back along the glass compression plates during recovery. In the absence of tack stress $\Phi=1$, and equation (5) is recovered.

Since the tack stress always resists slippage at the tendon surface, its sign during creep is the same as that of the creep stress. Therefore, from equation (A3), it causes $\Phi<1$ and hence increases the relative residual strain for any given recovery time $t-t_{\mathrm{u}}$. Equation (A3) also reveals that recovery will not continue indefinitely. The rate of recovery is predicted to reach zero at a time $t^{*}$, beyond which equation (A3) ceases to apply and the residual strain remains constant at its corresponding value $\left\langle\varepsilon_{2}\right\rangle_{y}^{*}$. By differentiation of equation (A3) this limiting condition may be found:

$$
t^{*}=\frac{t_{\mathrm{u}}}{1-\Phi^{1 /(1-n)}} \quad \text { and } \quad \frac{\left\langle\varepsilon_{2}\right\rangle_{y}^{*}}{\left\langle\varepsilon_{2}\right\rangle_{y}^{\max }}=\left[1-\Phi^{1 /(1-n)}\right]^{1-n}
$$

From knowledge of $n$ and the final relative residual strain, the second of equations (A4) allowed $\Phi$ to be determined. Hence, via the second of equations (A3), the magnitude of apparent tack stress $\left\langle\sigma_{1}\right\rangle_{x}$ was found for each creep/recovery test. 


\section{References}

[1] Almekinders L.C., Weinhold P.S., Maffulli N., 2003. “Compression etiology in tendinopathy", Clinics in Sports Medicine 22, pp. 703-710.

[2] Docking, S., Samiric, T., Scase, E., Purdam, C., Cook, J., 2013. "Relationship between compressive loading and ECM changes in tendons", Muscles, Ligaments and Tendons Journal 3, pp. 7-11.

[3] Cook, J.L., and Purdam, C., 2013. "Is compressive load a factor in the development of tendinopathy?" British Journal of Sports Medicine 46, pp. 163-168.

[4] LaPrade, C.M., Civitarese, D.M., Rasmussen, M.T., and LaPrade, R.F., 2015. "Emerging updates on the posterior cruciate ligament: a review of the current literature", American Journal of Sports Medicine 43, pp. 3077-3092.

[5] Middleton, K.K., Hamilton, T., Irrgang, J.J., Karlsson, J., Harner, C.D., and Fu, F.H., 2014. "Anatomic anterior cruciate ligament (ACL) reconstruction: a global perspective. Part 1", Knee Surgery, Sports Traumatology, Arthroscopy 22, pp. 1467-1482.

[6] Rigby, B.J., Hirai, N., Spikes, J.D., and Eyring, H., 1959. "The mechanical properties of rat tail tendon,” The Journal of General Physiology 43, pp. 265-283. 
[7] Cohen, R.E., Hooley, C.J., and McCrum, N.G., 1976. "Viscoelastic creep of collagenous tissue," Journal of Biomechanics 9, pp. 175-184.

[8] Hooley, C.J., McCrum, N.G., and Cohen, R.E., 1980. "The viscoelastic deformation of tendon," Journal of Biomechanics 13, pp. 521-528.

[9] Johnson, G.A., Tramaglini, D.M., Levine, R.E., Ohno, K., Choi, N-Y., and Woo, S.L-Y., 1994. "Tensile and viscoelastic properties of human patella tendon," The Journal of Bone and Joint Surgery 12, pp. 796-803.

[10] Duenwald, S. E., Vanderby, R., and Lakes, R.S., 2009. "Viscoelastic relaxation and recovery of tendon," Annals of Biomedical Engineering 37, pp. 1131-1140.

[11] Duenwald, S. E., Vanderby, R. and Lakes, R.S., 2010. "Stress relaxation and recovery in tendon and ligament: experiment and modelling," Biorheology 47, pp. 1-14.

[12] Dourte, L. M., Pathmanathan, L., Jawad, A.F., Iozzo, R.V., Mienaltowski, M.J., Birk, D.E., and Soslowsky, L.J., 2012. "Influence of decorin on the mechanical, compositional, and structural properties of the mouse patellar tendon," ASME Journal of Biomechanical Engineering 134, 031005 (8pp).

[13] Davis, F. M. and De Vita, R, 2012. "A nonlinear constitutive model for stress relaxation in ligaments and tendons", Annals of Biomedical Engineering 40, pp. 2541-2550. 
[14] LaCroix, A. S., Duenwald-Kuehl, S.E., Brickson, S., Akins, T.L., Diffee, G., Aiken, J., Vanderby, R., and Lakes, R.S., 2013. "The effect of age and exercise on the viscoelastic properties of rat tail tendon," Annals of Biomedical Engineering 41, pp. 1120-1128.

[15] Ikeda, J., Zhao, C., Chen, Q., Thoreson, A.R., An, K-N., and Amadio, P.C., 2011. "Compressive properties of cd-HA-gelatin modified intrasynovial tendon allograft in canine model in vivo," Journal of Biomechanics 44, pp. 1793-1796.

[16] Lee, S-B., Nakajima, T., Luo, Z-P., Zobitz, M.E., Chang, Y-W., and An, K-N., 2000. “The bursal and articular sides of the supraspinatus tendon have a different compressive stiffness," Clinical Biomechanics 15, pp. 241-247.

[17] Lynch, H. A., Johannessen, W., Wu, J.P., Jawa, A., and Elliott, D.M., 2003. "Effect of fiber orientation and strain rate on the nonlinear uniaxial tensile material properties of tendon," ASME Journal of Biomechanical Engineering 125, pp. 726-731.

[18] Williams, L. N., Elder, S.H., Bouvard, J.L., and Horstemeyer, M.E., 2008. "The anisotropic compressive mechanical properties of the rabbit patellar tendon,” Biorheology 45, pp. 577586. 
[19] Main, E. K., Goetz, J.E., Rudert, M.J., Goreham-Voss, C.M., and Brown, T.D., 2011. "Apparent transverse compressive material properties of the digital flexor tendons and the median nerve in the carpal tunnel," Journal of Biomechanics 44, pp. 863-868.

[20] Salisbury, S.T.S., Buckley, C.P., and Zavatsky, A.B., 2016. "Transverse compression of tendons,” ASME Journal of Biomechanical Engineering 138, 041002 (9 pp).

[21] Salisbury, S. T. S., Buckley, C.P., and Zavatsky, A.B., 2008. "Image-based non-contact method to measure cross-sectional areas and shapes of tendons and ligaments," Measurement Science and Technology 19, 045705 (9 pp).

[22] Chimich, D., Shrive, N., Frank, C., Marchuk, L., and Bray, R., 1992. "Water content alters viscoelastic behaviour of the normal adolescent rabbit medial collateral ligament," Journal of Biomechanics 25, pp. 831-837.

[23] Salisbury, S. T. S., D.Phil. thesis, University of Oxford, 2008.

[24] Fung, Y. C., 1993. Biomechanics: Mechanical Properties of Living Tissues, $2^{\text {nd }}$ edition. New York: Springer.

[25] Provenzano, P., Lakes, R., Keenan, T., and Vanderby, R., 2001. "Nonlinear ligament viscoelasticity,” Annals of Biomedical Engineering 29, pp. 908-914. 
[26] Elliott, D. M., Robinson, P.S., Gimbel, J.A., Sarver, J.J., Abboud, J.A., Iozzo, R.V., and Soslowsky, L.J., 2003. "Effect of altered matrix proteins on quasilinear viscoelastic properties in transgenic mouse tail tendons", Annals of Biomedical Engineering 31, pp. 599605.

[27] Buckley, C. P. and Jones, D.C., 1995. "Glass-rubber constitutive model for amorphous polymers near the glass transition," Polymer 36, pp. 3301-3312.

[28] Hingorami, R. V., Provenzano, P.P., Lakes, R.S., Escarcega, A., and Vanderby, R., 2004. "Nonlinear viscoelasticity in rabbit medial collateral ligament," Annals of Biomedical Engineering 32, pp. 306-312.

[29] Bonifasi-Lista, C., Small, S.P., and Weiss, J.A., 2005. "Viscoelastic properties of the human medial collateral ligament under longitudinal, transverse and shear loading," Journal of Orthopaedic Research 23, p. 67-76.

[30] Hannafin, J.A., and Arnoczky, S.P., 1994. "Effect of cyclic and static tensile loading on water content and solute diffusion in canine flexor tendons: an in vitro study", Journal of Orthopaedic Research 12, pp. 350-356.

[31] Han, S., Gemmell, S.J., Helmer, K.G., Grigg, P., Wellen, J.W., Hoffman, A.H., and Sotak, C.H., 2000. "Changes in ADC caused by tensile loading of rabbit Achilles tendon: evidence for water transport," Journal of Magnetic Resonance 144, pp. 217-227. 
[32] Haut, T. L., and Haut, R.C., 1997. "The state of tissue hydration determines the strain-ratesensitive stiffness of human patellar tendon," Journal of Biomechanics 30, pp. 79-81.

[33] Nordin, M., Lorenz, T., and Campello, M., 2001. "Biomechanics of ligaments and tendons", Chapter 4 in Basic Biomechanics of the Musculoskeletal System (3rd ed.), eds. Nordin, M. and Frankel, V.H., Lippincott Williams \& Wilkins (Philadelphia), pp. 102-125.

[34] Wellen, J., Helmer, K.G., Grigg, P., Sotak, C.H., 2004. “Application of porous-media theory to the investigation of water $\mathrm{ADC}$ changes in rabbit Achilles tendon caused by tensile loading”, Journal of Magnetic Resonance 170, pp. 49-55.

[35] Wellen, J., Helmer, K.G., Grigg, P. and Sotak, C.H., 2005. "Spatial characterization of $\mathrm{T}_{1}$ and $\mathrm{T}_{2}$ relaxation times and the water apparent diffusion coefficient in rabbit Achilles tendon subjected to tensile loading", Magnetic Resonance in Medicine 53, pp. 535-544.

[36] Ross, M.H. and Pawlina, W., 2011. “Connective tissues”, Chapter 6 in Histology: a text and atlas (6th ed.), Lippincott Williams \& Wilkins (Philadelphia), pp. 158-197.

[37] Creton, C., and Leibler, L., 1996. "How does tack depend on time of contact and contact pressure?” Journal of Polymer Science: Part B: Polymer Physics 34, pp. 545-554. 
Journal of Biomechanical Engineering

\section{Acknowledgements}

This work was supported in part by the Leverhulme Trust (UK). 


\section{Figure Captions}

Figure 1: The representative race-track cross-section shape, shown by Salisbury et al. [20] to approximate the shape of tendons after pre-conditioning in transverse compression. Along the axes of mirror symmetry $\mathrm{AB}$ and $\mathrm{CD}$, principal axes 1,2,3 are co-linear with co-ordinate axes $\mathrm{O} x, \mathrm{O} y, \mathrm{O} z$. The compression plates are shown hatched.

Figure 2: An example set of creep and recovery data for one of the tendons. Displacements are represented as the averaged strain $\left\langle\varepsilon_{2}\right\rangle_{y}$. Magnitudes of the corresponding applied creep stresses $\left\langle\sigma_{2}\right\rangle_{x}^{c}$ are as shown (in $\left.\mathrm{kPa}\right)$.

Figure 3: A double logarithmic plot of creep data (for clarity, only 1 in 200 of the data points are shown) for the same ten tests as shown in Figure 2. The lines through the data points are linear regressions, over creep times $10 \mathrm{~s}-100 \mathrm{~s}$.

Figure 4: Values of the coefficient $A$ in the power law expression for the averaged strain during creep (equations (3)), fitted to all creep curves (from $10 \mathrm{~s}-100 \mathrm{~s}$ ) for all seven tendons, plotted versus the magnitude of the applied creep stress: $-\left\langle\sigma_{2}\right\rangle_{x}^{c}$. Each symbol refers to a different tendon.

Figure 5: Values of the exponent $n$ in the power law expression for the averaged strain during creep (equations (3)), fitted to all creep curves (from $10 s-100 s$ ) for all seven tendons, plotted versus the magnitude of the applied creep stress: $-\left\langle\sigma_{2}\right\rangle_{x}^{c}$. Symbols refer to the same tendons as in 
Figure 4. Also shown (line) is an empirical curve least-squares fitted to the data points (equation (4)).

Figure 6: The relative residual strain remaining after a recovery time of $5 t_{\mathrm{u}}$, following creep tests of duration $t_{\mathrm{u}} \approx 120 \mathrm{~s}$, for all ten tests on each of the seven tendons, plotted versus the magnitude of the applied creep stress: $-\left\langle\sigma_{2}\right\rangle_{x}^{c}$. Symbols refer to the same tendons as in Figures 4 and 5.

Figure 7: Typical plots of the magnitude of 'tack stress' $-\left\langle\sigma_{1}\right\rangle_{x}^{c}$ versus the magnitude of applied creep stress: $-\left\langle\sigma_{2}\right\rangle_{x}^{c}$, for four tendons (symbols, referring to the same tendons as in Figures $4-$ 6 ), as deduced from the residual strains remaining at a recovery time $=5 t_{\mathrm{u}}$. Also shown (lines) are linear regressions through each set of points.

Figure 8: Typical plots of residual strain versus recovery time, for four magnitudes of applied creep stress shown (in kPa), for the tendon in Figures 2 and 3. Also shown (lines) are the corresponding displacements calculated from equations (A3). 


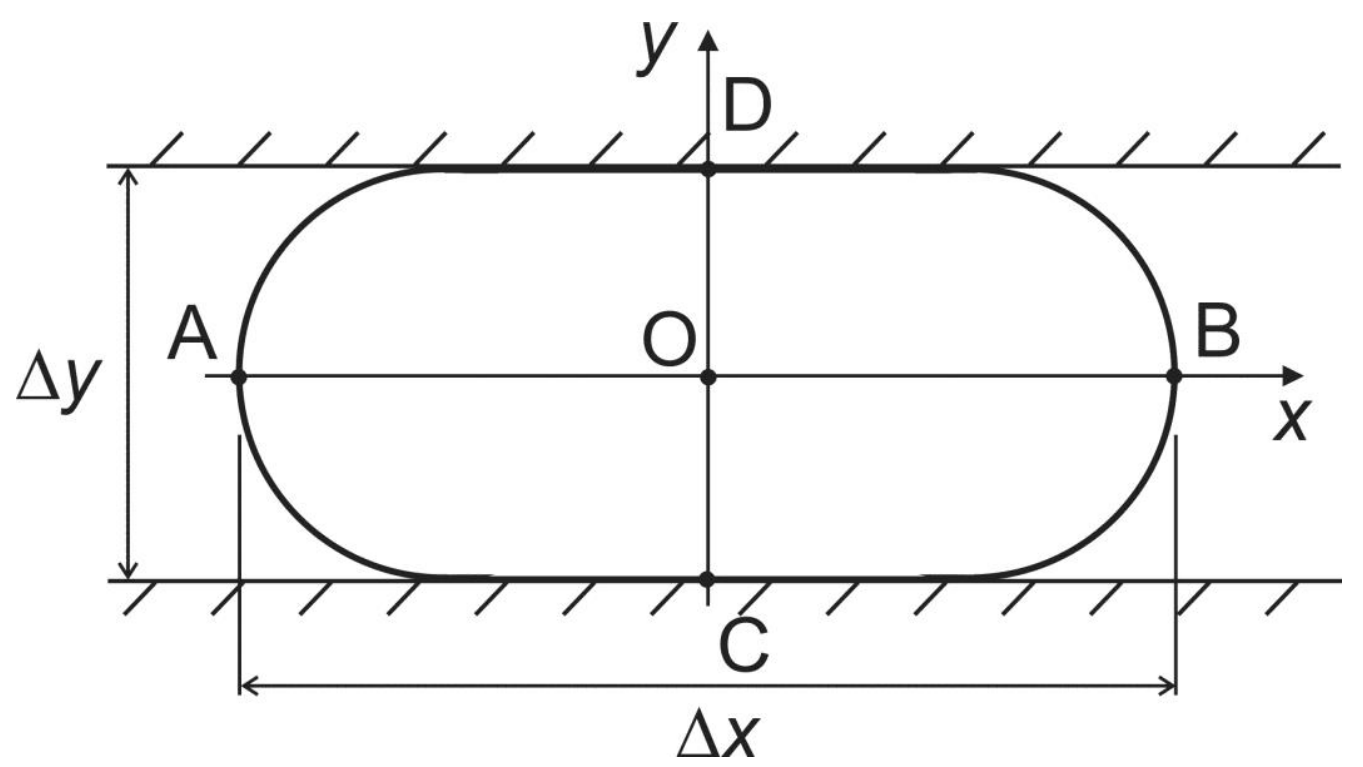

Figure 1. 


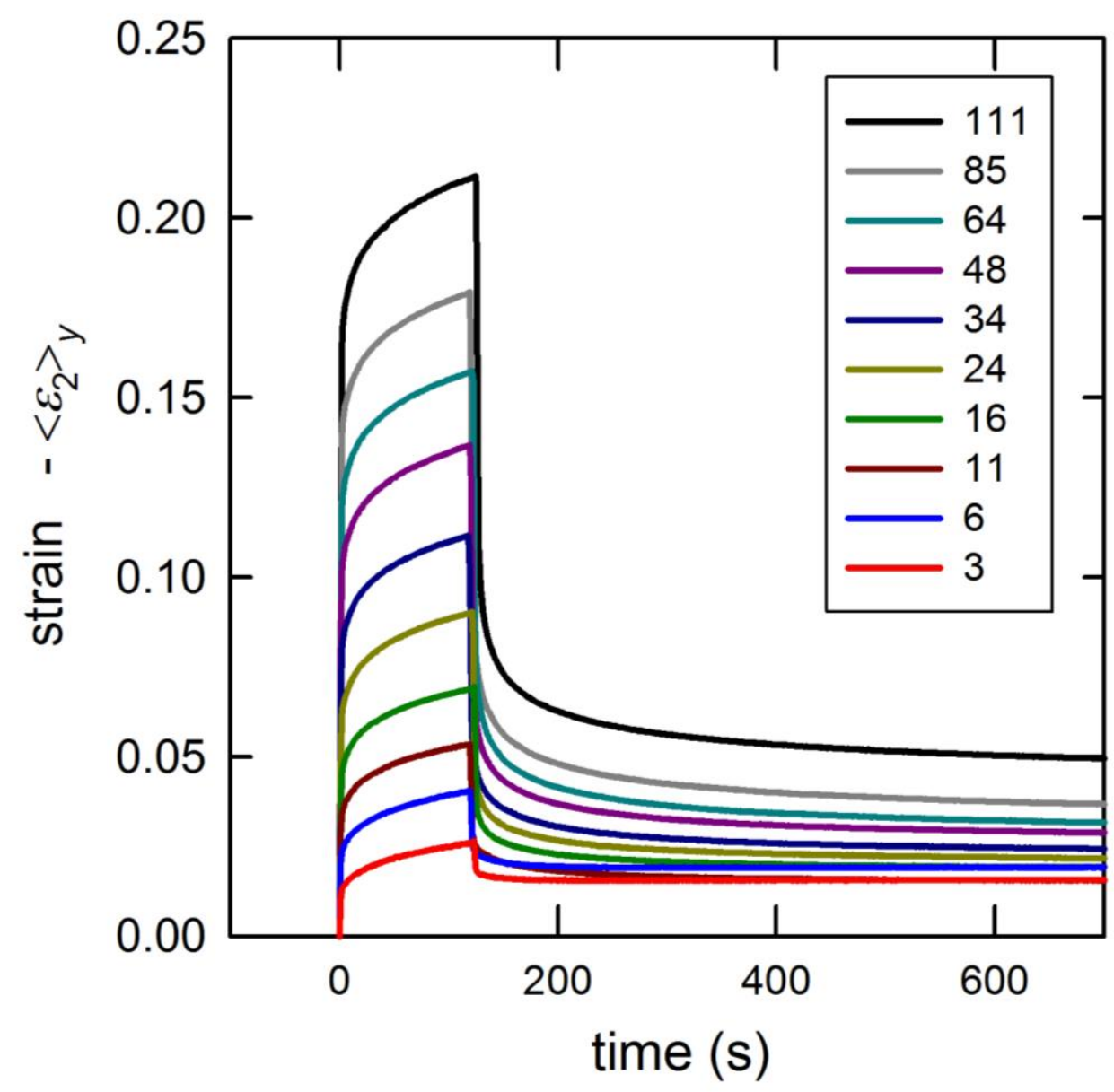

Figure 2. 


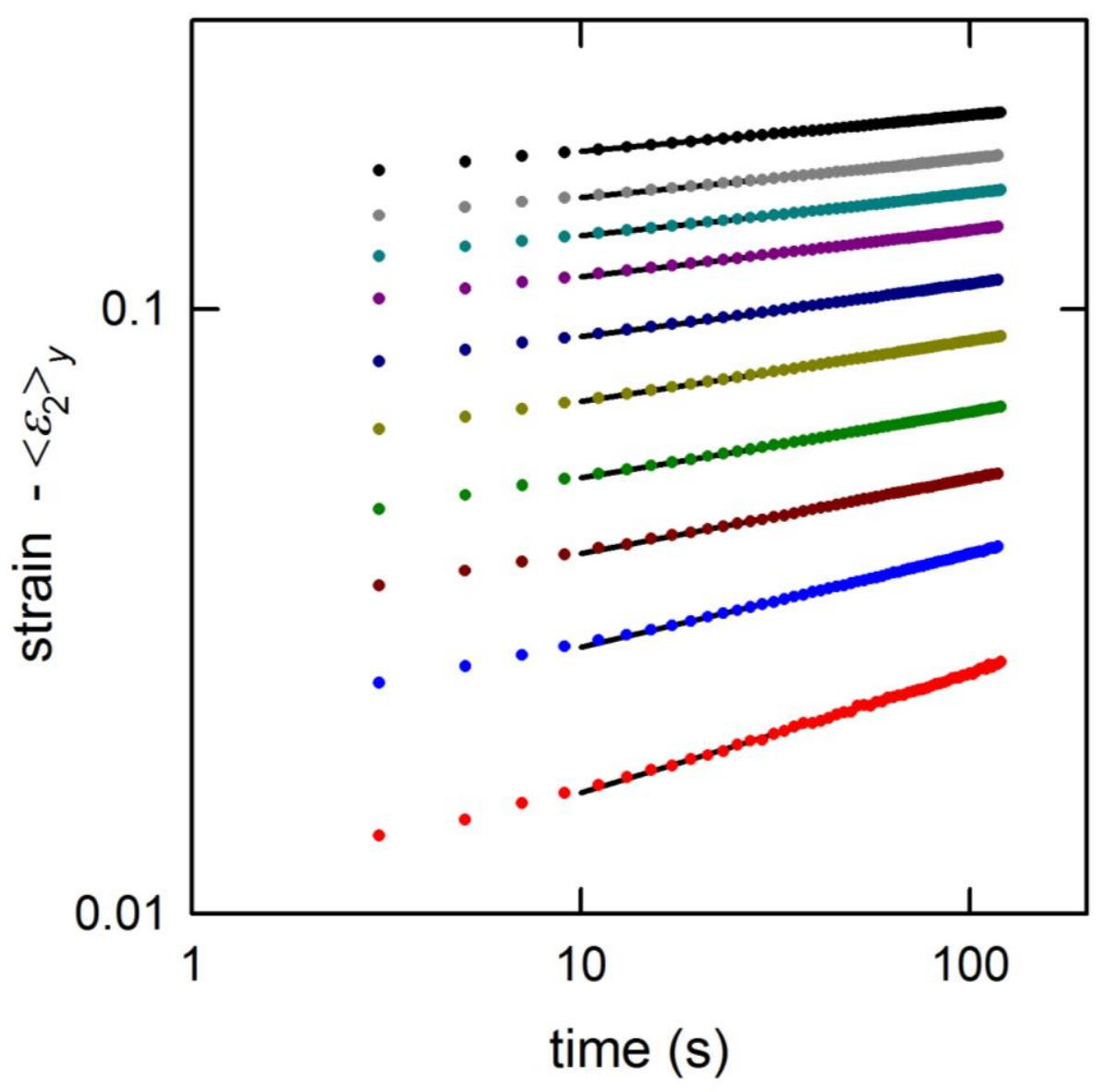

Figure 3. 


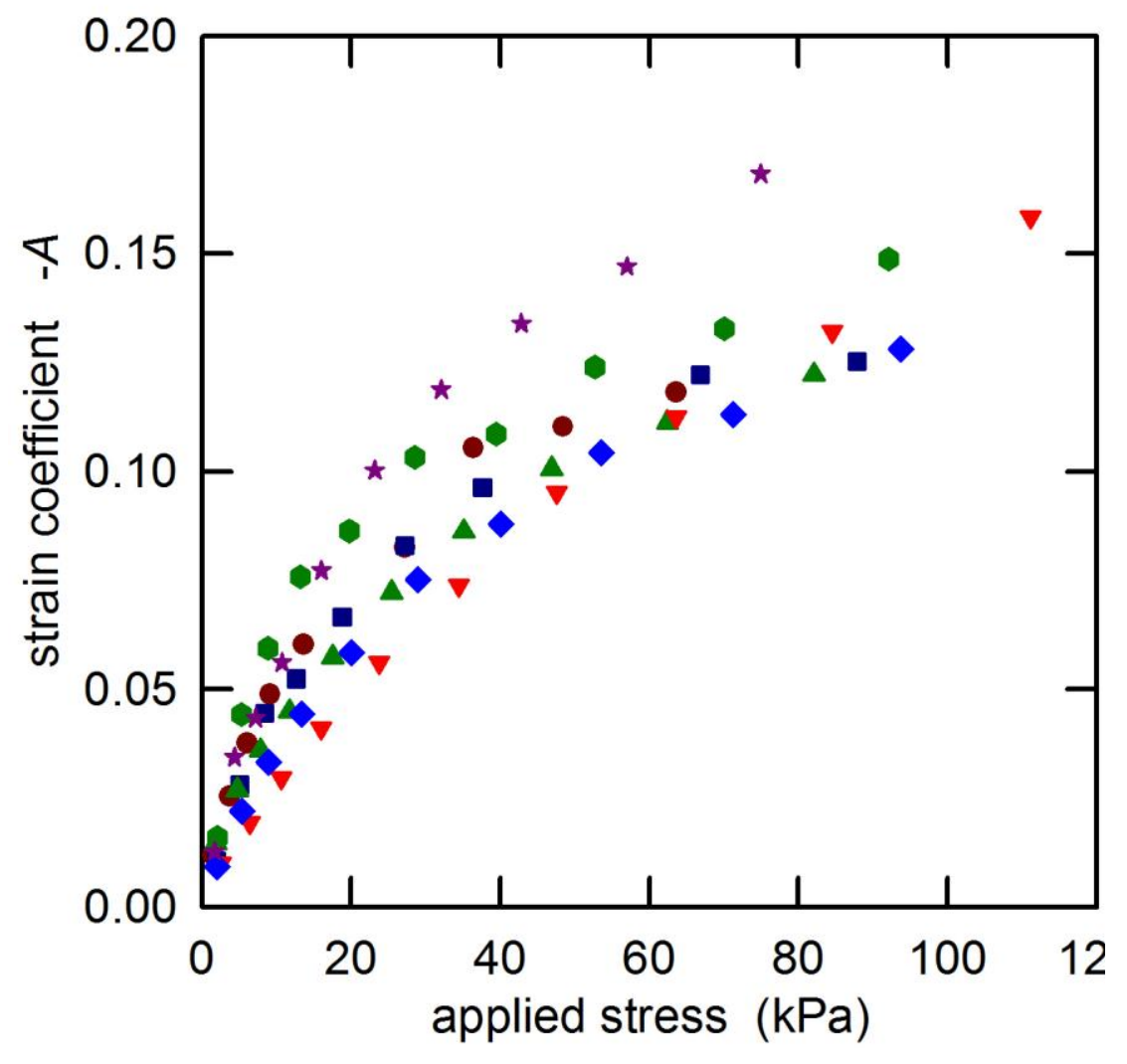

Figure 4. 


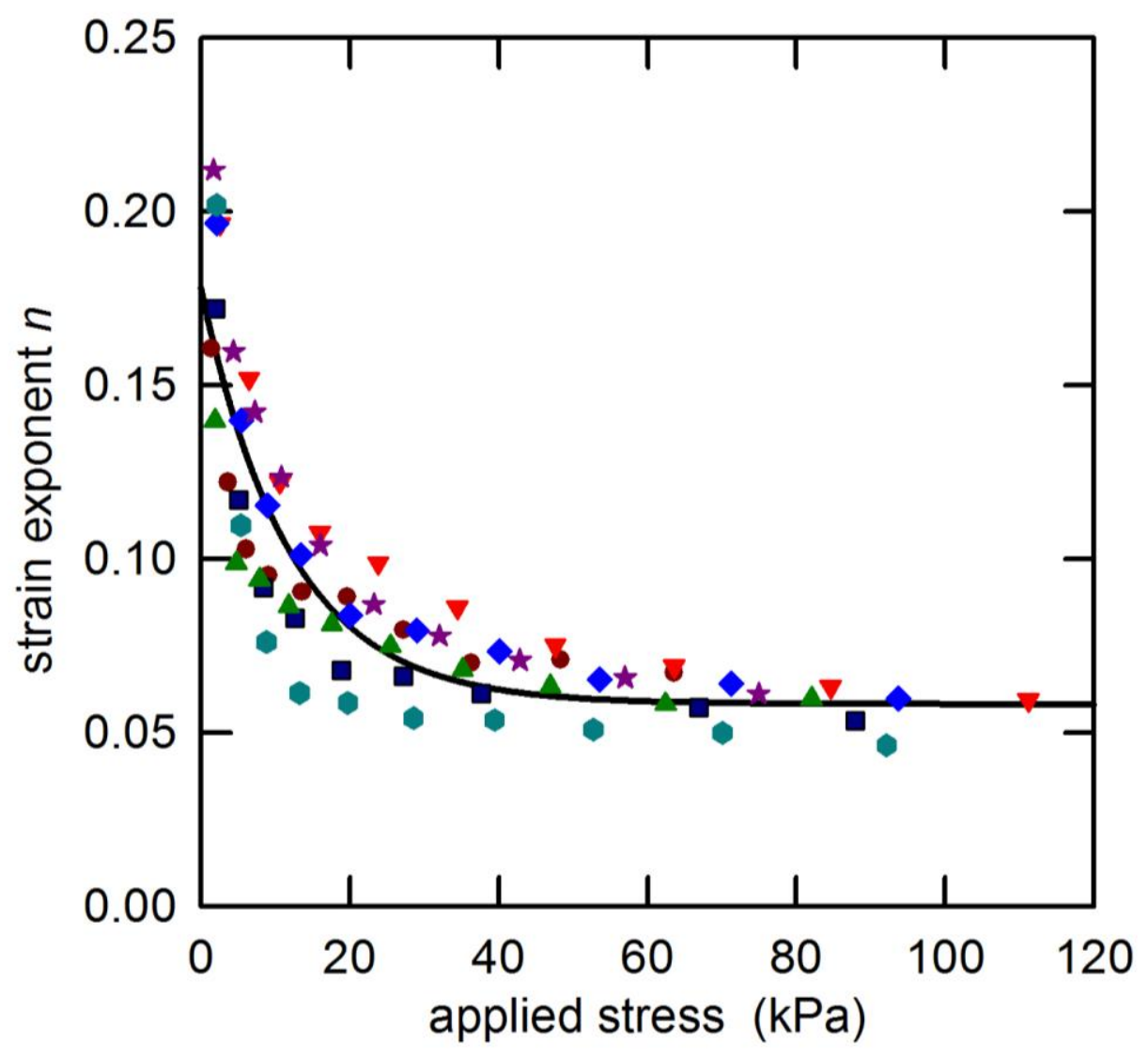

Figure 5. 


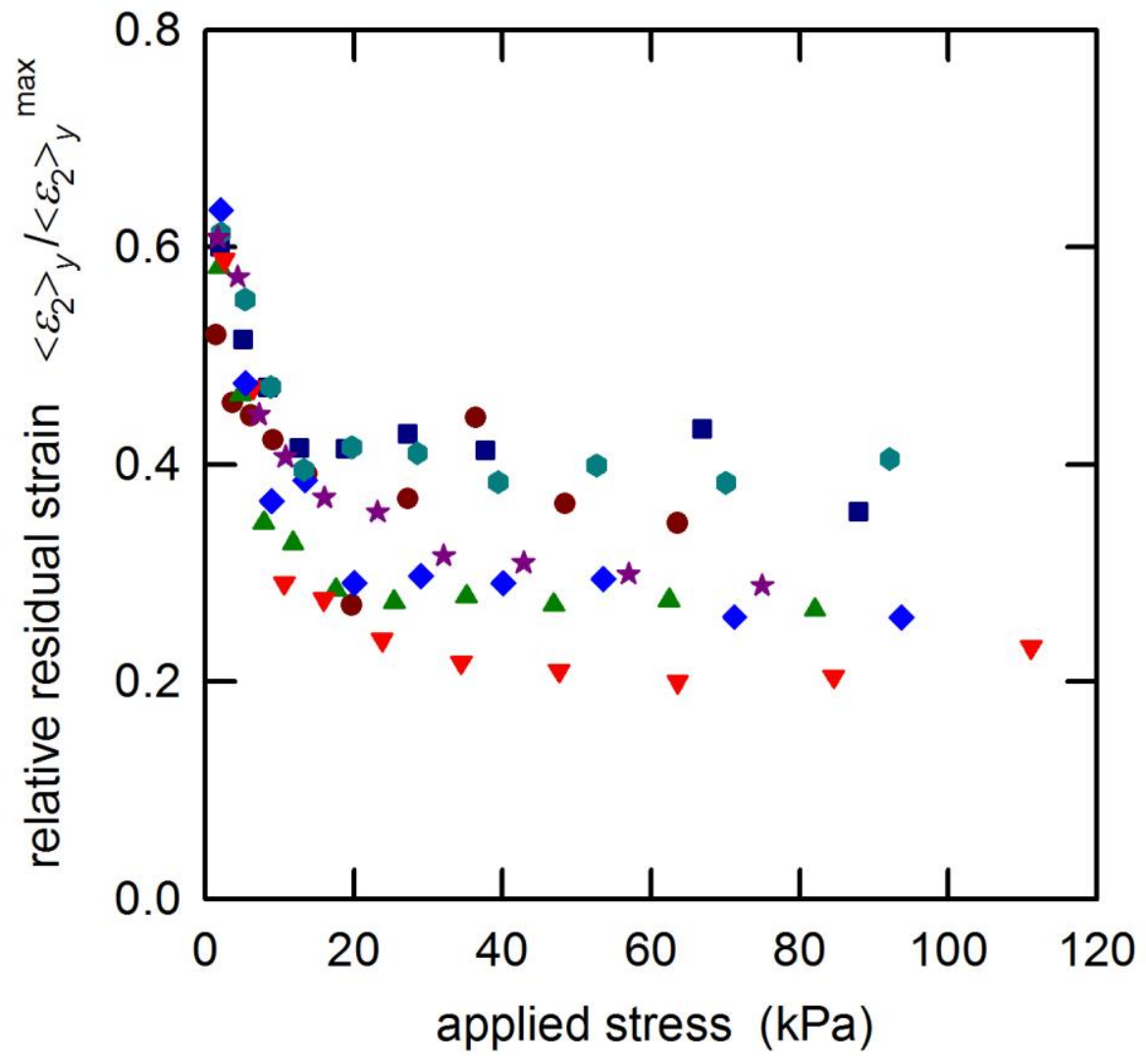

Figure 6. 


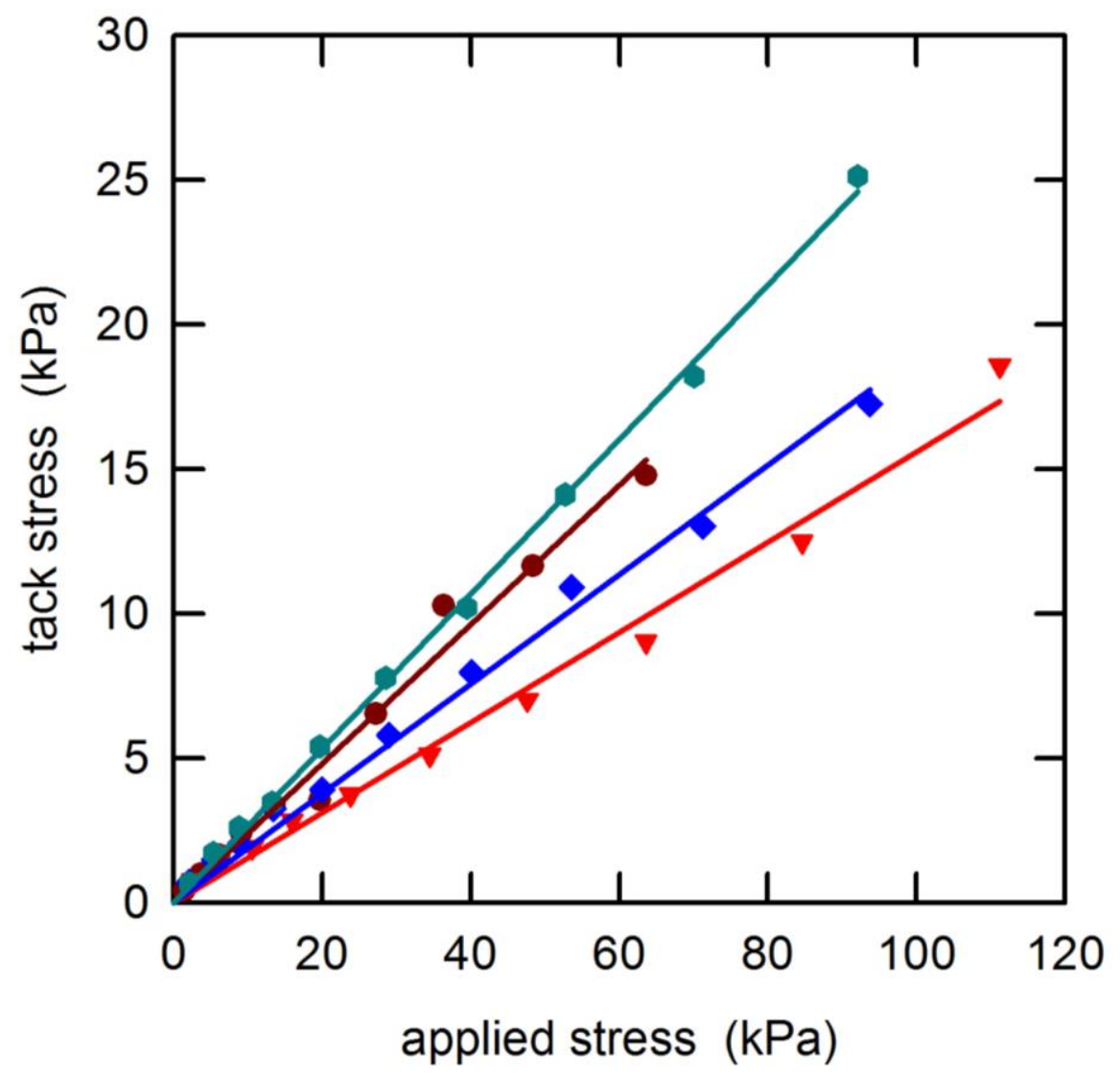

Figure 7. 


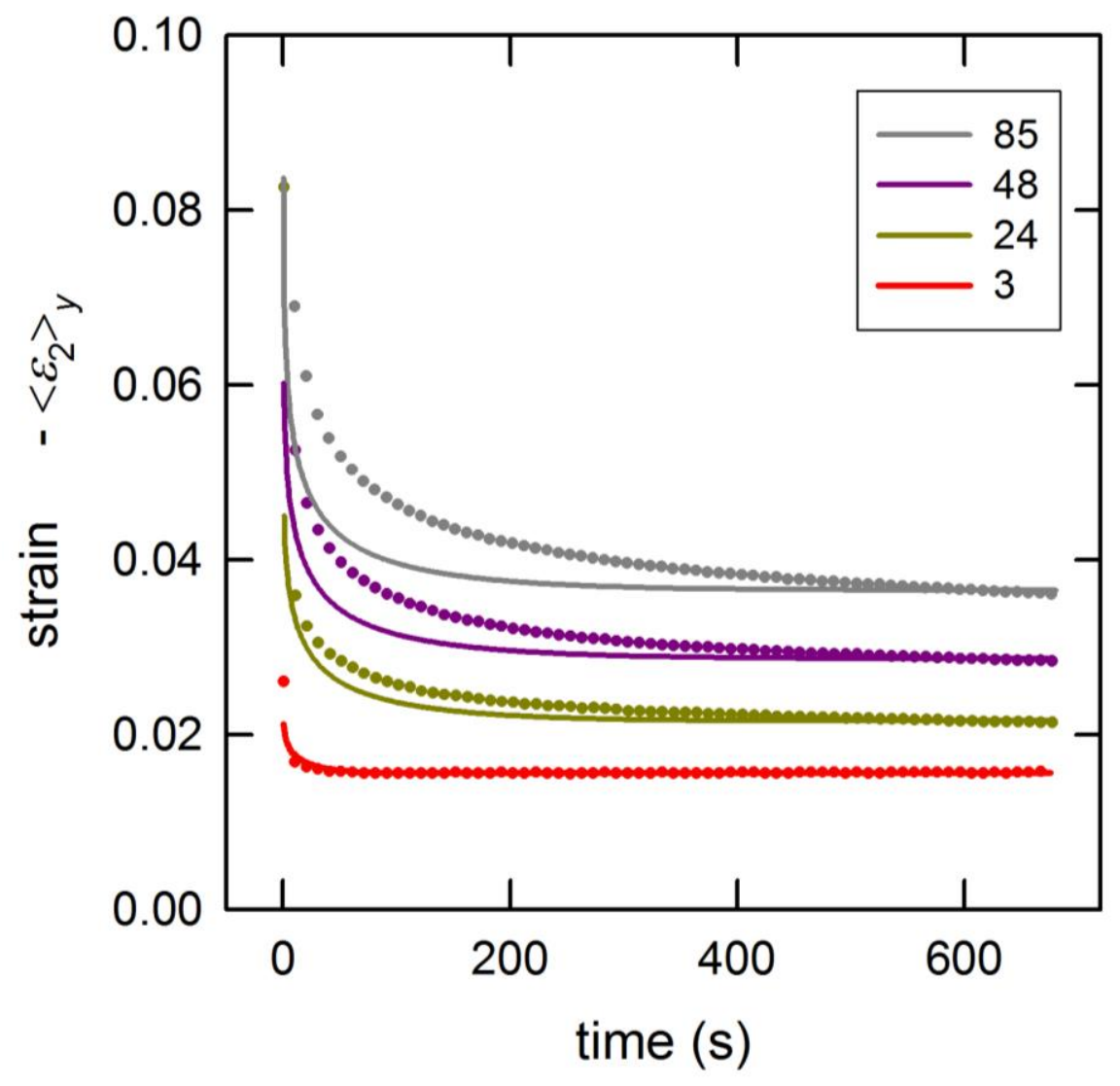

Figure 8. 